\title{
Radiological biomarkers for assessing response to locoregional therapies in hepatocellular carcinoma: From morphological to functional imaging (Review)
}

\author{
XIAO-DAN YE ${ }^{1}$, ZUGUO YUAN $^{2,3}$, JIAN ZHANG $^{4}$ and ZHENG YUAN ${ }^{5,6}$ \\ ${ }^{1}$ Department of Radiology, Shanghai Chest Hospital Affiliated to Shanghai Jiaotong University, Shanghai 200030; \\ ${ }^{2}$ Radiation Oncology Center, The 1st Affiliated Hospital of Zhejiang University, Hangzhou, Zhejiang 310000; \\ ${ }^{3}$ Department of Radiotherapy, Ningbo Yinzhou Hospital, Ningbo, Zhejiang 315100; ${ }^{4}$ Department of Nuclear Medicine, \\ Xinhua Hospital Affiliated to Shanghai Jiaotong University School of Medicine, Shanghai 200092; \\ ${ }^{5}$ Department of Radiology, Fudan University Shanghai Cancer Center, Shanghai 200032; \\ ${ }^{6}$ Department of Radiology, Shanghai 85 Hospital, Shanghai 200052, P.R. China
}

Received August 25, 2016; Accepted January 16, 2017

DOI: $10.3892 /$ or.2017.5420

\begin{abstract}
Many hepatocellular carcinoma (HCC) patients do not qualify for curative surgical intervention and are instead treated with locoregional therapies (LRTs) including ablative and endovascular therapies. Assessment of imaging response is essential in the management of HCC for determining efficacy of therapy and as a surrogate marker for improved survival. The established morphological image biomarkers for tumor burden measurement continue to be applied, as size measurement can easily be used in clinical practice. However, in the setting of liver-directed LRTs for HCC, simple tumor morphological changes can be less informative and usually appear later than biologic changes. Functional imaging (such as perfusion and diffusion imaging, PET-CT/MR and MR spectroscopy) has the potential to be a promising technique for assessment of HCC response to LRTs. Although promising, none of these functional imaging biomarkers have gone through all the required steps of standardization and validation and established accepted criteria for clinical practice.
\end{abstract}

\section{Contents}

1. Introduction

2. Morphological imaging response criteria (size-based) of HCC to LRT

3. Enhancement-based functional imaging response criteria of HCC to LRTs

Correspondence to: Dr Zheng Yuan, Department of Radiology, Shanghai 85 Hospital, 1328 Huashan Road, Shanghai 200052, P.R. China

E-mail: yuanzheng0404@163.com

Key words: hepatocellular carcinoma, response evaluation criteria, WHO criteria, RECIST, radiological biomarker, functional imaging, perfusion, diffusion, positron emission tomography, MR spectroscopy
4. Perfusion imaging for assessing response of HCC to LRTs

5. Diffusion-weighted MR imaging for assessing response of HCC to LRTs

6. Positron emission tomography (PET)/PET-computed tomography (PET-CT) imaging for assessing response of HCC to LRTs

7. Other novel functional imaging biomarkers for assessing response of HCC to LRTs

8. Conclusion

\section{Introduction}

Liver cancer is a highly prevalent disease worldwide and one of the leading causes of cancer death in the world (1). An estimated 782,500 new liver cancer cases and 745,500 deaths occurred worldwide during 2012, with China alone accounting for approximately $50 \%$ of the total number of cases and deaths. Despite the declining rate for liver cancer in China, population growth and ageing still led to a large and rising number of new cases in 2015 (2). Most (70-90\%) primary liver cancers occurring worldwide are hepatocellular carcinoma (HCC). Because of the presence of advanced disease or poor liver function, many HCC patients are not candidates for curative surgical treatments (resection or transplantation). These patients may be eligible for treatment with locoregional therapies (LRTs) including ablative and endovascular therapies, and/or with cytostatic targeted molecular systemic therapies such as sorafenib, which achieve some survival benefits for unresectable HCC (3). Accurately determining tumor response after therapy has become essential in the management of HCC for determining efficacy of therapy (4), subsequent therapeutic planning and as a surrogate marker for improved survival (5). Lack of objective response after one or more LRTs is associated with poor survival, although it may be influenced by the type of LRTs used (6-8) and pattern of tumor progression (intrahepatic or extrahepatic) (9).

Here, we review various proposed clinical response criteria of HCC to LRTs from morphological to functional imaging 


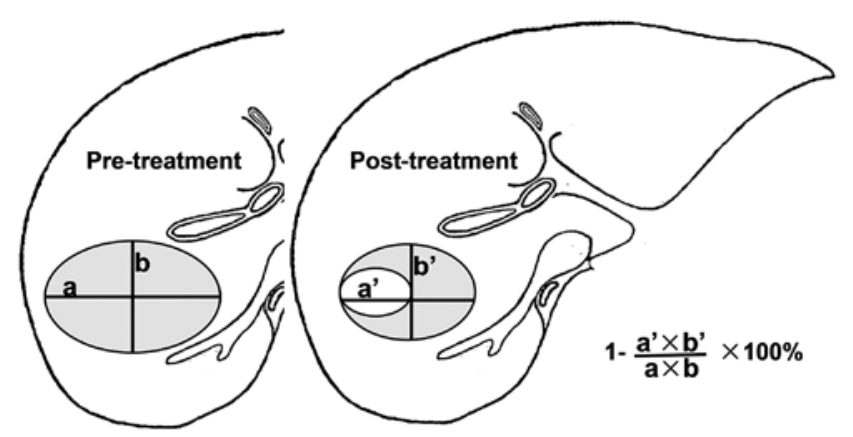

Figure 1. Illustration of the method of measurement according to the World Health Organization (WHO) response criteria (a, maximum diameter of entire tumor before treatment; $b$, diameter perpendicular to $a$; $a$ ', maximum diameter of entire smaller tumor after treatment; $b$ ', diameter perpendicular to a').

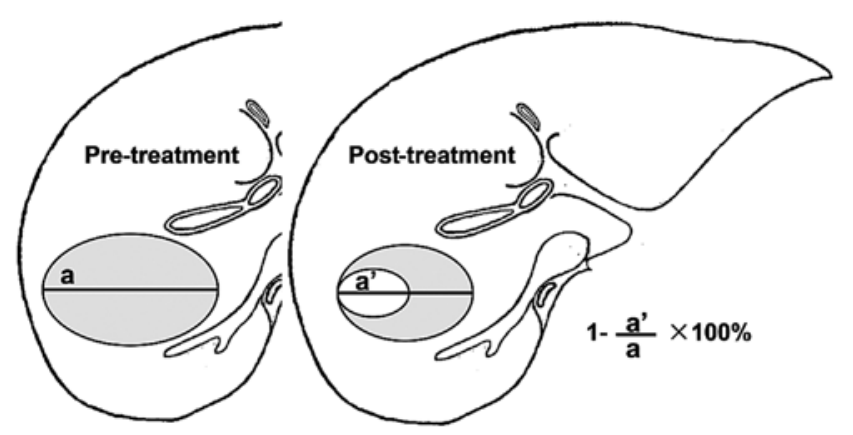

Figure 2. Illustration of the method of measurement according to the Response Evaluation Criteria in Solid Tumors (RECIST) (a, maximum diameter of entire tumor before treatment; a', maximum diameter of entire smaller tumor after treatment).

biomarkers, assess their accuracy for determining tumor response after LRTs and discuss their challenges in clinical practice.

\section{Morphological imaging response criteria (size-based) of HCC to LRT}

In 1981, the World Health Organization (WHO) proposed the first tumor response criterion after therapy (WHO criterion) (10) (Fig. 1). In 2000, the Response Evaluation Criteria in Solid Tumors 1.0 (RECIST version 1.0) was proposed (11) and updated (RECIST version 1.1) in 2009 (12) (Table I and Fig. 2), which addressed the shortcoming of the WHO criterion. The objective the WHO and RECIST response criteria assess overall tumor burden using morphological tumor-size measurements on imaging and require assessment at baseline and on follow-up imaging. Objective response assessment is based on morphological tumor-size change after therapy and classified into complete response (CR), partial response (PR), no change (NC) and progressive disease (PD) (Table I).

LRTs for HCC induce tumor necrosis and reduced vascularity. Some LRTs initially lead to even an increase in apparent tumor size due to extensive necrosis. This is a major limitation of morphological (size-based) imaging response criteria for assessing response of HCC to LRTs. Therefore, morphological (size-based) imaging response criteria for determination of therapeutic success that rely solely on change in tumor size after therapy may be inappropriate to HCC treated with LRTs (13).

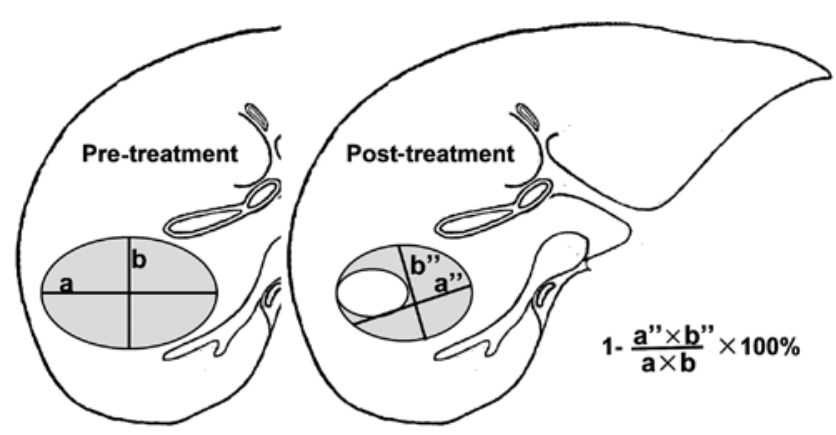

Figure 3. Illustration of the method of measurement according to the European Association for the Study of the Liver (EASL) criteria. The gray area within the tumor represents enhancing viable tumor tissue, and the white area represents non-enhancing lesions. (a, maximum diameter of entire tumor before treatment; $b$, diameter perpendicular to $a ; a "$, maximum diameter of enhancing larger area of tumor after treatment; $b^{\prime \prime}$, diameter perpendicular to $\left.a^{\prime \prime}\right)$.

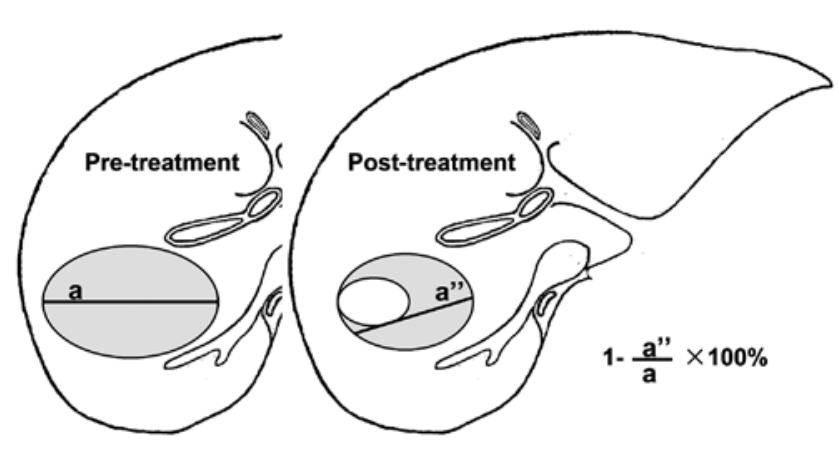

Figure 4. Illustration of the method of measurement according to the modified response evaluation criteria in solid tumors (mRECIST) criteria. The gray area within the tumor represents enhancing viable tumor tissue, and the white area represents non-enhancing lesions. (a, maximum diameter of entire tumor before treatment; a", maximum diameter of enhancing larger area of tumor after treatment).

In addition, more objective imaging response criteria that are specific to the therapy type have been developed (14-20).

In summary, for the determination of therapeutic success of HCC to LRTs, morphological (size-based) imaging measurements using WHO, RECIST 1.0 and RECIST 1.1 criteria may not be applicable because these therapies result in tumor necrosis regardless of change in size.

\section{Enhancement-based functional imaging response criteria of HCC to LRTs}

In 2000, The European Association for the Study of the Liver (EASL) proposed determination of therapeutic success of HCC to LRTs should measure the size of residual viable tumor rather than the overall size of the tumor (14) (Table I and Fig. 3). In 2005, the American Association for the Study of Liver Diseases (AASLD) guideline cited this concept (15). In 2010, the EASL published the modified response evaluation criteria in solid tumors (mRECIST) criteria for HCC response to LRTs based on measuring only the viable enhancing areas of the HCC after LRTs, excluding portion of necrosis. It is as an amendment and update to the AASLD-JNCI Expert Panel Criteria and are based on the most recent 2009 RECIST 1.1 and EASL 2000 criteria (16) (Table I and Fig. 4). The mRECIST criteria assesses HCC at baseline, after LRTs and 


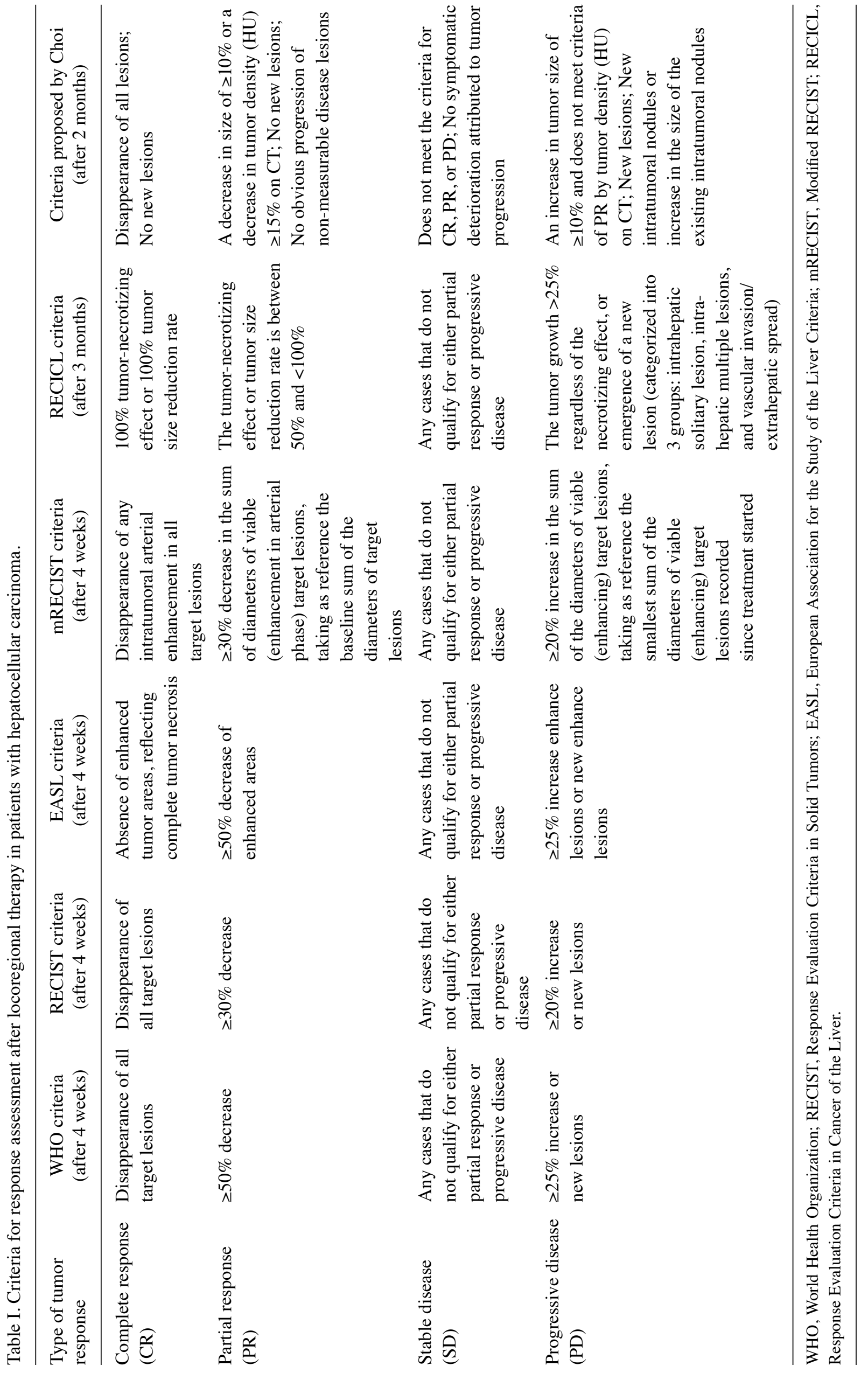


overall response. In the process of clinical application, high quality arterial-phase enhanced CT/MR imaging is required. There are also other response criteria for HCC response to LRTs based on enhancement CT/MRI exam. In 1994, the Liver Cancer Study Group of Japan used a similar system (RECICL) (17) to assess direct treatment effect and overall disease status after therapy, which was updated in 2004, and again in $2009(18,19)$. In 2007, Choi et al $(20)$ proposed the Choi Response Criteria for HCC response to antivascular therapy based on tumor density and volume.

Therefore, the EASL and the AASLD-JNCI and mRESIST criteria suggested assessing response criteria for HCC response to LRTs based on the size of viable enhancing tumor rather than overall tumor size including area of necrosis. These criteria have been shown to correlate well with histopathologic response than WHO and RECIST criteria. Previous studies of patients with HCC treated with ablation $(21,22)$, TACE $(6-8,21,23-26)$, and transarterial radioembolization (TARE) with Y90 $(24,25)$ have shown that mRECIST and EASL criteria have excellent intercriterion concordance and are more accurate at predicting complete histopathologic response (23) and survival after therapy than WHO and RECIST criteria (25).

First of all, a major shortcoming of enhancement-based models of imaging response systems for HCC response to LRTs is that they assess all target and non-target lesions in the entire liver without taking into consideration that different tumors in the same patient are not treated at the same time (27). Thus, the overall prognosis in such patients is unclearly determined by the behavior of the treated or untreated tumors. Recent studies have shown one or two primary target lesion responses to LRTs correlate well with disease progression and survival in patients with solitary and multifocal $\operatorname{HCC}(25,28,29)$. Additionally, the EASL and mRECIST criteria do not take into consideration the change of the overall tumor size after LRTs as an index of response, because several studies have shown the reduction in overall tumor size correlates well with long-term response $(25,30)$. Secondly, for enhancement-based models of imaging response systems for $\mathrm{HCC}$ response to LRTs, any single enhancement-based imaging technique may not be adequate for assessment of response to all types of LRTs (30). For example, all enhancement-based models of imaging response systems (except for the Choi Response Criteria) do not accurately reflect the result of therapies that result in decreased enhancement without frank necrosis, such as transarterial chemoembolization (TACE) (30).

In summary, enhancement-based systems of response to LRTs assessing change in size of viable enhancing tumor rather than overall tumor size have been shown to correlate well with histopathologic response and are more accurate at predicting survival after therapy than WHO and RECIST size-based models. Any single enhancement-based system may not be adequate for assessment of response to all types of LRTs. Of course, the three-dimensional volumetric tumor measurements could be more accurately identify the result of HCC response to LRTs, and it is a priority in future clinical trial research (16).

\section{Perfusion imaging for assessing response of HCC to LRTs}

Almost all enhancement-based models of imaging response systems (except for the Choi Response Criteria) do not accu- rately reflect the result of therapies that result in decreased enhancement without frank necrosis, such as conventional TACE or radioembolization (30). Furthermore, HCC exhibits local alterations in microvascular anatomy, demonstrating neoangiogenesis, promoted by vascular endothelial growth factor (VEGF). Antiangiogenic therapies have demonstrated promising results in HCC. The result of HCC treated with molecular targeted agents showed that they initially suppress tumor growth by downregulating angiogenesis and it is not sufficient to assess treatment response by measurement of tumor size or the residual viable tumor size (31). Since the therapeutic effects on the tumor microvascular environment alter tissue perfusion, physiologic imaging techniques such as dynamic contrast-enhanced ultrasound (D-CEUS), CT or MR perfusion imaging begins to play a critical role in the evaluation of therapies that result in decreased enhancement without necrosis $(32,33)$.

The fundamental principle of perfusion imaging is based on DCE imaging techniques that compute the temporal changes in tissue enhancement after intravenous administration of contrast media. A variety of imaging protocols have been proposed for perfusion imaging and the protocol selection should be made on the availability of the scanner technology and the pertinent physiologic parameter of interest. The computed perfusion parameters are dependent on the scan protocol and the mathematical model/software for image processing $(34,35)$. The commonly described perfusion CT parameters include blood flow (BF), blood volume (BV), permeability surface area (PS), time to peak enhancement (TTP) and transfer constant $\left(\mathrm{K}^{\text {trans }}\right)$. Similarly for perfusion $\mathrm{MR}$, transfer constant $\left(\mathrm{K}^{\text {trans }}\right)$ is the most accepted quantitative surrogate end point from compartment models $(36,37)$.

CEUS and D-CEUS were acknowledged to be a feasible examination for evaluating dynamic changes in tumor vascularity in patients with HCC undergoing antiangiogenic target therapy (38) and chemoembolization (39). Previously, HCC shows hyper-vascularised tumor enhancement type. After treatment, it shows lack of contrast enhancement, whereas still viable tumor shows arterial-enhancing and subsequent washout (40). CEUS and D-CEUS could be used to identify the result of $\mathrm{HCC}$ response to antiangiogenic therapies, predict tumor responses and patient survival (38). The times to peak intensity, mean transit time (MTT) values and area under the curve (AUC) levels, correlated well with tumor responses and survival rates (32). In addition, AUC, time to peak intensity and slope of wash-in were positively associated with progression free survival (PFS) (32). In fact, CEUS and D-CEUS are low cost and good safety examinations can provide both morphological and functional data. In addition, its important role in clinical application has been recently highlighted by EFSUMB guidelines (41). For example this panel of experts recognised the important role of CEUS in the very early evaluation of ablative treatment as a guidance for immediate retreatment of residual unablated tumor (41). More recently, three-dimensional CEUS technique (3D CEUS) has been reported to improve the study of tumor vascularity, thus, allowing the response evaluation of HCC treatments in the three orthogonal planes. Nevertheless, 3D CEUS may be limited by the spatial resolution of the current $3 \mathrm{D}$ probes in the assessment of therapeutic response of HCC treated with 
ablative treatments compared to conventional CEUS (42). Additionally, the best timing and the best quantitative dynamic parameters for the assessment HCC response to LRTs are still unclear.

On perfusion CT, HCC has been reported to show substantially higher perfusion (high BF, BV and PS with low MTT) compared to normal liver tissue (43). After antiangiogenic drugs or HCC directed therapies, decrease in tumor perfusion parameters has been shown within days of initiation of treatment $(43,44)$. Similarly, Zhu et al $(43)$ have shown that HCC nodules showing more substantial reduction in tumor permeability $\left(\mathrm{K}^{\text {trans }}\right)$ on perfusion MR soon after sunitinib, had better long-term outcome. Liang et al (45) reported Signal parameters of DCE-MRI over tumor and liver parenchyma correlated with tumor response and survival, respectively, in HCC patients receiving radiotherapy combination with an anti-angiogenic agent.

With CT, relatively high radiation dose and limited coverage of the anatomy are two major drawbacks of perfusion technique. Several efforts are being made with low dose scanning approaches (46). Likewise, there is no consensus on a scanning protocol or a mathematical model specific for HCC. Since the liver has a dual arterial and portal venous perfusion, the scan protocols should ideally include dual inputs to estimate quantitative perfusion parameters for hepatic tumors. However, due to larger tumor burden in advanced HCC and frequent occurrence of angioinvasion into the portal venous system, single arterial input is often applied as a simplifying assumption (47). More recently, volume perfusion CT (VPCT) enables quantification of perfusion in tumor tissue in absolute values by measuring flow and concentration of iodinated contrast medium during a time period within blood vessels and tissue generating time density curves (TDC) (48). This technique is also designed to calculate separately hepatic arterial and portal venous blood flow to the liver and liver tumors based on input functions obtained by regions of interest (ROIs) set in the spleen and the portal vein, the former representing a substitute for direct hepatic arterial measurements. VPCT have been used to characterize HCC (49) and monitor the HCC response to TACE and analysis of TACE-impact on tumor and uninvolved liver parenchymal perfusion at day one postTACE (50).

MR imaging has several advantages over CT, including the lack of ionizing radiation. Therefore, it has the ability to image whole organs repeatedly and dynamically with high temporal resolution, and the possibility of repeating the study multiple times after treatment. A variety of imaging protocols, other than DCE imaging technique after intravenous gadolinium contrast, have been proposed for perfusion MR imaging. Transcatheter intraarterial perfusion (TRIP) MR imaging involves direct catheter-based intraarterial injection of contrast material (51), which offers a functional alternative to conventional digital subtraction angiography in the assessment of tumor perfusion changes during TACE (52). Recently, unenhanced MR perfusion imaging using the arterial spin labeling (ASL) technique was also introduced to quantify perfusion in the liver (53). This perfusion method, which does not require the use of contrast media, is a non-invasive MR perfusion technique that may offer great potential as an alternative imaging method for pure liver portal perfusion (53).
There are several drawbacks in liver perfusion MRI technique. General challenges confronting perfusion MR include lack of accepted standards of image acquisition and analysis, variable reproducibility and no established response evaluation criteria. Furthermore, unlike the linear relationship between iodine concentration and Hounsfield units on CT, the relationship between gadolinium concentration and signal intensity (SI) is non-linear with MR imaging, complicating quantitative perfusion measurements.

In summary, the possibility of evaluating tissue vascularization through perfusion imaging has led to the exploration of these imaging techniques as new assessment tools in order to measure the effectiveness of intraarterial therapy with or without antiangiogenic therapies for HCC. Different imaging biomarkers for assessing response to therapy in HCC derive from different imaging technique and the protocol selection should be made on the availability of the scanner technology and the pertinent physiologic parameter of interest. Further studies are warranted to determine the still unclear aspects such as the best timing and the best quantitative dynamic parameter for the assessment of response to HCC treatment.

\section{Diffusion-weighted MR imaging for assessing response of HCC to LRTs}

Diffusion-weighted MR imaging (DWI) has the unique ability of being able to provide information that reflects tissue cellularity and cellular membrane integrity (54). Moreover, apparent diffusion coefficient (ADC) measurement on an ADC map can be quantified by acquiring images with a different gradient duration and amplitude (i.e., b-value). DWI and ADC maps reflect the water molecule diffusion in tissue and can discriminate viable tumor from necrotic tissue. Viable tumor cells have intact membranes that restrict water molecules, whereas necrotic tissue shows increased water molecule diffusion as a result of cell membrane disruption (55). This makes it an attractive and useful technique for the assessment of tumor response after LRTs in patients with HCC.

The visual assessment of DWI, which includes images at higher b-values ( $\geq 500 \mathrm{sec} / \mathrm{mm}^{2}$ ), may aid to distinguish the different components of HCC (viable and necrotic components) following LRTs. As a general observation, necrotic HCC tissues (liquefaction or coagulation necrosis) secondary to LRTs typically show lower signal intensity on higher b-value images than viable tissues. ADC has also been used for early evaluation and prediction response to LRTs (56-64). An increase in ADC values has been reported following radioembolization $(56,57)$ and chemoembolization $(58-62)$ in the early post-treatment period (a few days up to 2 weeks) with measurable differences before and after treatment (58-62), but the treatment effect was noted 1-3 months after treatment.

The role of the pretreatment ADC value in predicting the response to LRTs have also been investigated with discordant results $(58,65)$, which may be related to the nature of tumors with or without necrotic tissue before treatment (66). Recent studies have also shown that pre-treatment ADC values as well as changes in ADC values after treatment may provide useful information for predicting survival for patients with unresectable hepatocellular carcinoma $(63,67-69)$. Vandecaveye et al (63) reported that 1-month response deter- 
A

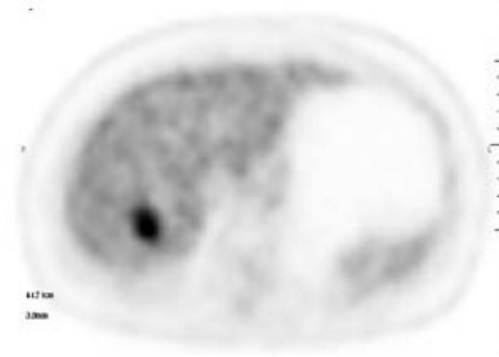

D

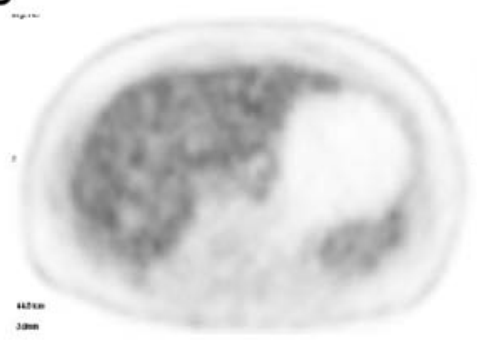

B

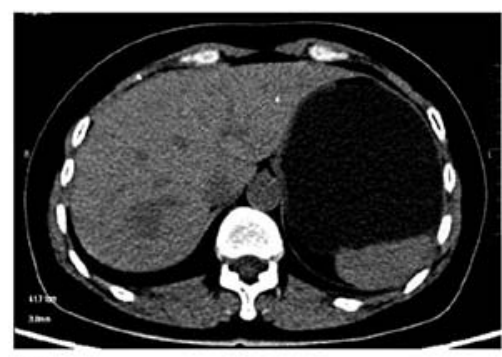

E

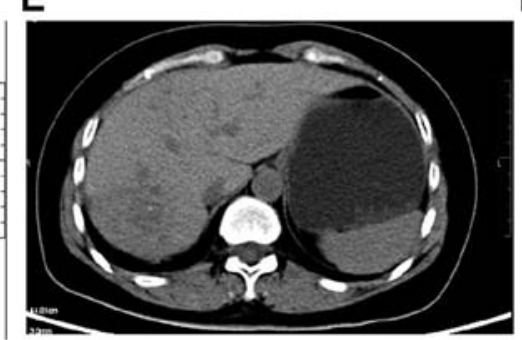

C

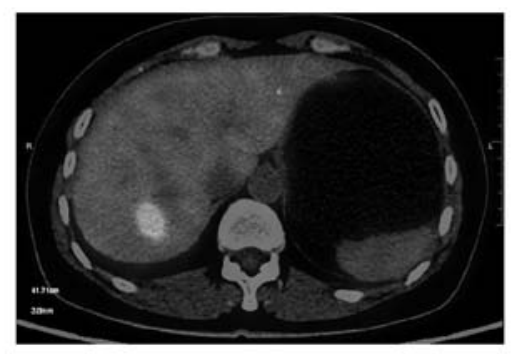

$\mathbf{F}$

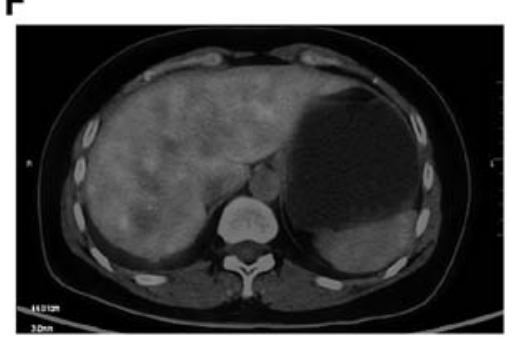

Figure 5. Baseline and follow-up PET-CT images of hepatocellular carcinoma (HCC). A 50-year-old female with HCC underwent microwave ablative and external beam radiotherapy. There was high tumor uptake in the first PET scan (before treatment, A, PET; B, CT; C, PET/CT fused axial images) and no uptake in the follow-up scan after therapy (D, PET; E, CT; F, PET/CT fused axial images). The patient belonged to the PET response group, indicating a good prognosis.

mined with apparent diffusion coefficient is an independent predictor of outcome for HCC treated with chemoembolization. Several studies have also shown that the pretreatment ADC values of liver malignancy can be a predictive factor of tumor response to RFA therapy (70); Mori et al (70) reported that the signal intensity of HCC on the ADC map was strongly associated with outcome after RFA. Hypointensity on the ADC map was the strongest independent factor related to recurrence and survival after RFA, even for small HCC (70).

In summary, previous studies of DWI in monitoring HCC response to LRTs, have uniformly reported increasing ADC during therapy onset proceeding anatomic size changes (71). DWI should be recommended as a routine method for evaluation of HCC response to LRTs, however, not in substitution but rather in combination with enhancement (EASL, mRECIST) criteria (72), because at this point, this technology is evolving with no accepted protocols and quantified standards, although the National Cancer Institute (NCI) has recognized the potential of this technique and has proposed consensus guidelines for DWI to meet minimum standards for its use as an effective image biomarker (73). In patients with contraindication to contrast agents or with slight-enhancement lesions, DWI can be considered a reasonable alternative to enhancement (EASL, mRECIST) criteria. However, further technological improvements (i.e., intravoxel incoherent motion diffusion-weighted MRI with bi-exponential diffusion model) and technique standardization are still required to use DWI at its full potential (74-76).

\section{Positron emission tomography (PET)/PET-computed tomography (PET-CT) imaging for assessing response of HCC to LRTs}

PET is a quantitative imaging modality and ${ }^{18} \mathrm{~F}$-fluorodeoxyglucose $\left({ }^{18} \mathrm{~F}-\mathrm{FDG}\right)$, a glucose analog, is the most commonly used PET tracer in clinical practice. ${ }^{18} \mathrm{~F}-\mathrm{FDG}-\mathrm{PET}$ has been considered to be a very useful non-invasive tool for diagnosis, tumor staging and monitoring of treatment responses in various malignancies (77). Recent studies have shown that PET-CT is useful in assessing HCC characterization. Low ${ }^{18} \mathrm{~F}$-FDG uptake is seen in well-differentiated HCC, whereas high ${ }^{18} \mathrm{~F}$-FDG uptake is observed in moderately to poorly differentiated HCC (78). Overall, the FDG-PET sensitivity in detecting HCC is lower (50-70\%) than other liver tumors. Standardized uptake value (SUV) is the accepted semi-quantitative biomarker of tracer uptake in PET. There is growing evidence that in PET-positive HCC, early metabolic response may reflect molecular changes and predict longterm outcome after completion of therapy (79-84). A SUV on ${ }^{18} \mathrm{~F}$-FDG PET-CT imaging can serve as an independent prognostic factor in $\mathrm{HCC}$ and may predict tumor recurrence after TACE $(79,83) .{ }^{18} \mathrm{~F}-\mathrm{FDG}$ uptake was an independent prognostic factor for PFS and OS in HCC patients treated with TACE or concurrent intra-arterial chemotherapy with external beam radiotherapy (CCRT). Especially, in HCCs with high ${ }^{18}$ F-FDG uptake, patients treated with CCRT showed better survival than those treated with TACE (Fig. 5). ${ }^{18} \mathrm{~F}-\mathrm{FDG}$ PET-CT may help determine the treatment modality for intermediate-to-advanced stage HCCs (80). An early interim PET-CT after TACE may have prognostic value for HCC patients treated with TACE and radiotherapy $(83,84)$. Therefore, the European Organization for Research and Treatment of Cancer (EORTC) has defined response assessment criteria for PET (85). EORTC has also suggested that the initial region of interest for SUV measurements should contain only viable tumors and be used consistently on the subsequent scans. To overcome some of the limitations of the EORTC criteria, Wahl et al (86) even proposed modified criteria with more stringent requirements for tumor response assessment with PET. 
In summary, PET and PET-CT have proven value in the imaging-based diagnosis of recurrent disease following LRTs of liver malignancies, and repeat treatment is often initiated solely based on this imaging modality. ${ }^{18} \mathrm{~F}-\mathrm{FDG}$ is not a tumorspecific tracer and the reproducibility of SUV is influenced by the time of image acquisition from tracer injection. Given the limitations of FDG in HCC, other tracers with different molecules including choline-based tracers are being investigated (87).

\section{Other novel functional imaging biomarkers for assessing response of HCC to LRTs}

MR spectroscopy (MRS) is a promising non-invasive technology. Hydrogen-1 $\left({ }^{1} \mathrm{H}\right)$ and phosphorus-31 $\left({ }^{31} \mathrm{P}\right) \mathrm{MRS}$ are the most common in vivo used in clinic. ${ }^{1} \mathrm{H}$-MRS has been used in the assessment of the effectiveness of chemoembolization treatment for HCC (88-91). The mean cho/lipid ratios were significantly decreased after TACE in HCC. Choline levels decreased significantly after treatment (88). The lipid value after TACE was proportionally increased because the iodized oil used for TACE has a substantial lipoid component (88). The studies of ${ }^{31} \mathrm{P}-\mathrm{MRS}$ assessing the effectiveness of chemoembolization treatment for HCC have shown that a significant decrease in nucleoside triphosphates (NTP) and an increase in Pi had been observed in the early phase of chemotherapy or chemoembolization in liver tumors (representing necrosis of tumor cells), followed by changes in phosphodiesters (PDE) and phosphomonoester (PME) levels $(92,93)$. Decreases in the $\mathrm{PDE} / \mathrm{NTP}$ ratio and the PDE/TPC ratio that occurred after treatment were the most remarkable changes secondary to chemoembolization. In the responsive group, the PDE/TPC ratio was significantly decreased after chemoembolization, whereas the NTP/TPC ratio was significantly increased. In the non-responsive group, phosphorus metabolism had no significant changes after treatment (94). There are several technical limitations in the assessment of the effectiveness of for HCC by MRS. This technology is evolving with no accepted protocols and quantified standards. Image distortions and susceptibility artifacts were unavoidable to some degree. New imaging sequences also need to develop to improve image quality (95).

Integrated PET-MRI is a new imaging modality, combining the advantages of FDG-PET with the ability of MRI to detect small liver tumors without $\mathrm{CT}$ radiation exposure. Recent results show that detection sensitivity for hepatic metastases, through post hoc fusion of FDG-PET images and 1.5 Tesla contrast-enhanced (Gadolinium) MRI obtained from two different scanners, is significantly higher than for PET-CT (96). PET-MRI appears to offer higher lesion conspicuity and diagnostic confidence compared to PET-CT (97), and this additional information can influence clinical management of cancer patients. The combined advantages of detection of smaller hepatic tumors with a higher sensitivity and detection of focal FDG uptake suggestive for local tumor progression indicates that PET-MRI could provide complementary information and facilitate improved clinical decision making (98). FDG PET-MRI could potentially improve the accuracy of (early) detection of progressive disease, and thus, allow swifter and more effective decision-making regarding appropriate treatment (99).

\section{Conclusion}

It is important that early and accurate assessment of the efficacy of HCC following intra-arterial (i.e., chemoembolization and radioembolization) and ablative (i.e., radiofrequency ablation and cryoablation) therapies in making therapeutic decisions, such as whether to repeat, interrupt or completely terminate therapy. Functional imaging has an important position in assessing tumor response in locoregional therapy for HCCs, which induce biologic changes that may be detected by functional imaging much earlier than morphological imaging. An ideal imaging biomarker should be able to detect an immediate response to any therapeutic regimen in one examination. Although promising, none of these functional imaging biomarkers have gone through all the required steps of standardization and validation and established accepted criteria for clinical practice. At present in clinical practice, different imaging biomarkers for assessing response to therapy in HCC derive from different imaging technique and the protocol selection should be made on the availability of the scanner technology and the pertinent physiologic parameter of interest. Therefore, it is unlikely to be the sole functional imaging biomarker of HCC response after LRTs. A combination of enhancement (EASL, mRECIST) criteria and functional imaging has been stated to be better in assessing therapeutic response of HCC after LRTs and in providing more information to guide future therapy.

\section{Acknowledgements}

This study was supported by the National Natural Science Foundation of China (No. 81301262, 81571629 and 81301218).

\section{References}

1. Torre LA, Bray F, Siegel RL, Ferlay J, Lortet-Tieulent J and Jemal A: Global cancer statistics, 2012. CA Cancer J Clin 65: 87-108, 2015

2. Chen W, Zheng R, Baade PD, Zhang S, Zeng H, Bray F, Jemal A, Yu XQ and He J: Cancer statistics in China, 2015. CA Cancer J Clin 66: 115-132, 2016.

3. Forner A, Llovet JM and Bruix J: Hepatocellular carcinoma. Lancet 379: 1245-1255, 2012.

4. Yaghmai V, Besa C, Kim E, Gatlin JL, Siddiqui NA and Taouli B: Imaging assessment of hepatocellular carcinoma response to locoregional and systemic therapy. AJR Am J Roentgenol 201: 80-96, 2013.

5. Lewandowski RJ, Mulcahy MF, Kulik LM, Riaz A, Ryu RK, Baker TB, Ibrahim SM, Abecassis MI, Miller FH, Sato KT, et al: Chemoembolization for hepatocellular carcinoma: Comprehensive imaging and survival analysis in a 172-patient cohort. Radiology 255: 955-965, 2010.

6. Yeo DM, Choi JI, Lee YJ, Park MY, Chun HJ and Lee HG: Comparison of RECIST, mRECIST, and choi criteria for early response evaluation of hepatocellular carcinoma after transarterial chemoembolization using drug-eluting beads. J Comput Assist Tomogr 38: 391-397, 2014.

7. Gillmore R, Stuart S, Kirkwood A, Hameeduddin A, Woodward N, Burroughs AK and Meyer T: EASL and mRECIST responses are independent prognostic factors for survival in hepatocellular cancer patients treated with transarterial embolization. J Hepatol 55: 1309-1316, 2011.

8. Prajapati HJ, Spivey JR, Hanish SI, El-Rayes BF, Kauh JS, Chen Z and Kim HS: mRECIST and EASL responses at early time point by contrast-enhanced dynamic MRI predict survival in patients with unresectable hepatocellular carcinoma (HCC) treated by doxorubicin drug-eluting beads transarterial chemoembolization (DEB TACE). Ann Oncol 24: 965-973, 2013. 
9. Reig M, Rimola J, Torres F, Darnell A, Rodriguez-Lope C, Forner A Llarch N, Ríos J, Ayuso C and Bruix J: Postprogression survival of patients with advanced hepatocellular carcinoma: Rationale for second-line trial design. Hepatology 58: 2023-2031, 2013.

10. Miller AB, Hoogstraten B, Staquet $M$ and Winkler A: Reporting results of cancer treatment. Cancer 47: 207-214, 1981.

11. Therasse P, Arbuck SG, Eisenhauer EA, Wanders J, Kaplan RS, Rubinstein L, Verweij J, Van Glabbeke M, van Oosterom AT, Christian MC, et al: New guidelines to evaluate the response to treatment in solid tumors. European Organization for Research and Treatment of Cancer, National Cancer Institute of the United States, National Cancer Institute of Canada. J Natl Cancer Inst 92: 205-216, 2000.

12. Eisenhauer EA, Therasse P, Bogaerts J, Schwartz LH, Sargent D, Ford R, Dancey J, Arbuck S, Gwyther S, Mooney M, et al: New response evaluation criteria in solid tumours: Revised RECIST guideline (version 1.1). Eur J Cancer 45: 228-247, 2009.

13. Bruix J and Sherman M; American Association for the Study of Liver Diseases: Management of hepatocellular carcinoma: An update. Hepatology 53: 1020-1022, 2011.

14. Bruix J, Sherman M, Llovet JM, Beaugrand M, Lencioni R, Burroughs AK, Christensen E, Pagliaro L, Colombo M and Rodés J; EASL Panel of Experts on HCC; European Association for the Study of the Liver: Clinical management of hepatocellular carcinoma. Conclusions of the Barcelona-2000 EASL conference. J Hepatol 35: 421-430, 2001.

15. Bruix J and Sherman M; Practice Guidelines Committee, American Association for the Study of Liver Diseases: Management of hepatocellular carcinoma. Hepatology 42: 1208-1236, 2005.

16. Lencioni R and Llovet JM: Modified RECIST (mRECIST) assessment for hepatocellular carcinoma. Semin Liver Dis 30 52-60, 2010.

17. Khalili K, Kim TK, Jang HJ, Yazdi LK, Guindi $M$ and Sherman M: Indeterminate 1-2-cm nodules found on hepatocellular carcinoma surveillance: Biopsy for all, some, or none? Hepatology 54: 2048-2054, 2011.

18. Kudo M, Kubo S, Takayasu K, Sakamoto M, Tanaka M, Ikai I, Furuse J, Nakamura K and Makuuchi M; Liver Cancer Study Group of Japan (Committee for Response Evaluation Criteria in Cancer of the Liver, Liver Cancer Study Group of Japan): Response Evaluation Criteria in Cancer of the Liver (RECICL) proposed by the Liver Cancer Study Group of Japan (2009 Revised Version). Hepatol Res 40: 686-692, 2010.

19. Kudo M, Ueshima K, Kubo S, Sakamoto M, Tanaka M, Ikai I, Furuse J, Murakami T, Kadoya M and Kokudo N; Liver Cancer Study Group of Japan: Response Evaluation Criteria in Cancer of the Liver (RECICL) (2015 Revised version). Hepatol Res 46: 3-9, 2016.

20. Choi H, Charnsangavej C, Faria SC, Macapinlac HA, Burgess MA, Patel SR, Chen LL, Podoloff DA and Benjamin RS: Correlation of computed tomography and positron emission tomography in patients with metastatic gastrointestinal stromal tumor treated at a single institution with imatinib mesylate: Proposal of new computed tomography response criteria. J Clin Oncol 25: 1753-1759, 2007.

21. Forner A, Ayuso C, Varela M, Rimola J, Hessheimer AJ, de Lope CR, Reig M, Bianchi L, Llovet JM and Bruix J: Evaluation of tumor response after locoregional therapies in hepatocellular carcinoma: Are response evaluation criteria in solid tumors reliable? Cancer 115: 616-623, 2009.

22. Li H, Guo Z, Si T and Wang H: EASL and mRECIST responses are independent predictors of survival in hepatocellular carcinoma patients treated with cryoablation. Eur J Gastroenterol Hepatol 25: 620-627, 2013.

23. Bargellini I, Vignali C, Cioni R, Petruzzi P, Cicorelli A, Campani D, De Simone P, Filipponi F and Bartolozzi C: Hepatocellular carcinoma: CT for tumor response after transarterial chemoembolization in patients exceeding Milan criteria - selection parameter for liver transplantation. Radiology 255: 289-300, 2010

24 Memon K, Kulik L, Lewandowski RJ, Wang E, Riaz A, Ryu RK, Sato KT, Marshall K, Gupta R, Nikolaidis P, et al: Radiographic response to locoregional therapy in hepatocellular carcinoma predicts patient survival times. Gastroenterology 141: 526-535.e2., 2011.

25. Riaz A, Miller FH, Kulik LM, Nikolaidis P, Yaghmai V, Lewandowski RJ, Mulcahy MF, Ryu RK, Sato KT, Gupta R, et al: Imaging response in the primary index lesion and clinical outcomes following transarterial locoregional therapy for hepatocellular carcinoma. JAMA 303: 1062-1069, 2010.
26. Shim JH, Lee HC, Kim SO, Shin YM, Kim KM, Lim YS and Suh DJ: Which response criteria best help predict survival of patients with hepatocellular carcinoma following chemoembolization? A validation study of old and new models. Radiology 262: 708-718, 2012.

27. Salem R, Miller FH, Yaghmai V and Lewandowski RJ: Response assessment methodologies in hepatocellular carcinoma: Complexities in the era of local and systemic treatments. J Hepatol 58: 1260-1262, 2013

28. Kim BK, Kim SU, Kim MJ, Kim KA, Kim DY, Park JY, Ahn SH, Han KH and Chon CY: Number of target lesions for EASL and modified RECIST to predict survivals in hepatocellular carcinoma treated with chemoembolization. Clin Cancer Res 19: 1503-1511, 2013.

29. Shim JH, Lee HC, Won HJ, Shin YM, Kim KM, Lim YS and Suh DJ: Maximum number of target lesions required to measure responses to transarterial chemoembolization using the enhancement criteria in patients with intrahepatic hepatocellular carcinoma. J Hepatol 56: 406-411, 2012.

30. Riaz A, Memon K, Miller FH, Nikolaidis P, Kulik LM, Lewandowski RJ, Ryu RK, Sato KT, Gates VL, Mulcahy MF, et al: Role of the EASL, RECIST, and WHO response guidelines alone or in combination for hepatocellular carcinoma: Radiologic-pathologic correlation. J Hepatol 54: 695-704, 2011.

31. Iwazawa J, Ohue S, Hashimoto N, Yasumasa K, Abe H and Mitani T: Bevacizumab-induced hypovascular hepatocellular carcinoma treated by transarterial chemoembolization in a patient with metastatic colon cancer. J Vasc Interv Radiol 21: 412-414, 2010.

32. Zocco MA, Garcovich M, Lupascu A, Di Stasio E, Roccarina D, Annicchiarico BE, Riccardi L, Ainora ME, Ponziani F, Caracciolo G, et al: Early prediction of response to sorafenib in patients with advanced hepatocellular carcinoma: The role of dynamic contrast enhanced ultrasound. J Hepatol 59: 1014-1021, 2013.

33. Roccarina D, Garcovich M, Ainora ME, Riccardi L, Pompili M, Gasbarrini A and Zocco MA: Usefulness of contrast enhanced ultrasound in monitoring therapeutic response after hepatocellular carcinoma treatment. World J Hepatol 7: 1866-1874, 2015.

34. Miles KA, Hayball MP and Dixon AK: Functional images of hepatic perfusion obtained with dynamic CT. Radiology 188: 405-411, 1993.

35. Kambadakone AR and Sahani DV: Body perfusion CT: Technique, clinical applications, and advances. Radiol Clin North Am 47: 161-178, 2009.

36. Morgan B, Thomas AL, Drevs J, Hennig J, Buchert M, Jivan A, Horsfield MA, Mross K, Ball HA, Lee L, et al: Dynamic contrastenhanced magnetic resonance imaging as a biomarker for the pharmacological response of PTK787/ZK 222584, an inhibitor of the vascular endothelial growth factor receptor tyrosine kinases, in patients with advanced colorectal cancer and liver metastases: Results from two phase I studies. J Clin Oncol 21: 3955-3964, 2003.

37. van Laarhoven HW, Rijpkema M, Punt CJ, Ruers TJ, Hendriks JC, Barentsz JO and Heerschap A: Method for quantitation of dynamic MRI contrast agent uptake in colorectal liver metastases. J Magn Reson Imaging 18: 315-320, 2003.

38. Lassau N, Koscielny S, Chami L, Chebil M, Benatsou B, Roche A, Ducreux M, Malka D and Boige V: Advanced hepatocellular carcinoma: Early evaluation of response to bevacizumab therapy at dynamic contrast-enhanced US with quantification-preliminary results. Radiology 258: 291-300, 2011.

39. Shaw CM, Eisenbrey JR, Lyshchik A, O'Kane PL, Merton DA, Machado P, Pino L, Brown DB and Forsberg F: Contrast-enhanced ultrasound evaluation of residual blood flow to hepatocellular carcinoma after treatment with transarterial chemoembolization using drug-eluting beads: A prospective study. J Ultrasound Med 34: 859-867, 2015.

40. Marcus CD, Ladam-Marcus V, Cucu C, Bouché O, Lucas L and Hoeffel $\mathrm{C}$ : Imaging techniques to evaluate the response to treatment in oncology: Current standards and perspectives. Crit Rev Oncol Hematol 72: 217-238, 2009.

41. Claudon M, Dietrich CF, Choi BI, Cosgrove DO, Kudo M, Nolsøe CP, Piscaglia F, Wilson SR, Barr RG, Chammas MC, et al; World Federation for Ultrasound in Medicine; European Federation of Societies for Ultrasound: Guidelines and good clinical practice recommendations for Contrast Enhanced Ultrasound (CEUS) in the liver - update 2012: A WFUMBEFSUMB initiative in cooperation with representatives of AFSUMB, AIUM, ASUM, FLAUS and ICUS. Ultrasound Med Biol 39: 187-210, 2013. 
42. Bartolotta TV, Taibbi A, Matranga D, Midiri M and Lagalla R: $3 D$ versus $2 D$ contrast-enhanced sonography in the evaluation of therapeutic response of hepatocellular carcinoma after locoregional therapies: Preliminary findings. Radiol Med (Torino) 120 695-704, 2015.

43. Zhu AX, Holalkere NS, Muzikansky A, Horgan K and Sahani DV: Early antiangiogenic activity of bevacizumab evaluated by computed tomography perfusion scan in patients with advanced hepatocellular carcinoma. Oncologist 13: 120-125, 2008.

44. Cheng AL, Kang YK, Chen Z, Tsao CJ, Qin S, Kim JS, Luo R, Feng J, Ye S, Yang TS, et al: Efficacy and safety of sorafenib in patients in the Asia-Pacific region with advanced hepatocellular carcinoma: A phase III randomised, double-blind, placebocontrolled trial. Lancet Oncol 10: 25-34, 2009.

45. Liang PC, Ch'ang HJ, Hsu C, Chen LT, Shih TT and Liu TW: Perfusion parameters of dynamic contrast-enhanced magnetic resonance imaging predict outcomes of hepatocellular carcinoma receiving radiotherapy with or without thalidomide. Hepatol Int 9: 258-268, 2015.

46. Griffiths JR, Tate AR, Howe FA and Stubbs M; Group on MRS Application to Cancer: Magnetic Resonance Spectroscopy of cancer-practicalities of multi-centre trials and early results in non-Hodgkin's lymphoma. Eur J Cancer 38: 2085-2093, 2002.

47. Schwarz AJ, Maisey NR, Collins DJ, Cunningham D, Huddart R and Leach MO: Early in vivo detection of metabolic response: A pilot study of 1H MR spectroscopy in extracranial lymphoma and germ cell tumours. Br J Radiol 75: 959-966, 2002.

48. Bota S, Piscaglia F, Marinelli S, Pecorelli A, Terzi E and Bolondi L: Comparison of international guidelines for noninvasive diagnosis of hepatocellular carcinoma. Liver Cancer 1: 190-200, 2012

49. Kaufmann S, Horger T, Oelker A, Kloth C, Nikolaou K, Schulze $\mathrm{M}$ and Horger M: Characterization of hepatocellular carcinoma (HCC) lesions using a novel CT-based volume perfusion (VPCT) technique. Eur J Radiol 84: 1029-1035, 2015.

50. Kaufmann S, Horger T, Oelker A, Beck S, Schulze M, Nikolaou K Ketelsen D and Horger M: Volume perfusion computed tomography (VPCT)-based evaluation of response to TACE using two different sized drug eluting beads in patients with nonresectable hepatocellular carcinoma: Impact on tumor and liver parenchymal vascularisation. Eur J Radiol 84: 2548-2554, 2015.

51. Wang D, Bangash AK, Rhee TK, Woloschak GE, Paunesku T, Salem R, Omary RA and Larson AC: Liver tumors: Monitoring embolization in rabbits with VX2 tumors - transcatheter intraarterial first-pass perfusion MR imaging. Radiology 245: 130-139, 2007.

52. Larson AC, Wang D, Atassi B, SatoKT, Ryu RK, Lewandowski RJ, Nemcek AA Jr, Mulcahy MF, Kulik LM, Miller FH, et al: Transcatheter intraarterial perfusion: MR monitoring of chemoembolization for hepatocellular carcinoma - feasibility of initial clinical translation. Radiology 246: 964-971, 2008.

53. Katada Y, Shukuya T, Kawashima M, Nozaki M, Imai H, Natori T and Tamano M: A comparative study between arterial spin labeling and CT perfusion methods on hepatic portal venous flow. Jpn J Radiol 30: 863-869, 2012.

54. Koh DM and Collins DJ: Diffusion-weighted MRI in the body: Applications and challenges in oncology. AJR Am J Roentgenol 188: 1622-1635, 2007.

55. Youn BJ, Chung JW, Son KR, Kim HC, Jae HJ, Lee JM, Song IC Kim IO and Park JH: Diffusion-weighted MR: Therapeutic evaluation after chemoembolization of VX-2 carcinoma implanted in rabbit liver. Acad Radiol 15: 593-600, 2008.

56. Deng J, Miller FH, Rhee TK, Sato KT, Mulcahy MF, Kulik LM, Salem R, Omary RA and Larson AC: Diffusion-weighted MR imaging for determination of hepatocellular carcinoma response to yttrium-90 radioembolization. J Vasc Interv Radiol 17: 1195-1200, 2006.

57. Kamel IR, Reyes DK, Liapi E, Bluemke DA and Geschwind JF: Functional MR imaging assessment of tumor response after $90 \mathrm{Y}$ microsphere treatment in patients with unresectable hepatocellular carcinoma. J Vasc Interv Radiol 18: 49-56, 2007.

58. Yuan Z, Ye XD, Dong S, Xu LC, Xu XY, Liu SY and Xiao XS: Role of magnetic resonance diffusion-weighted imaging in evaluating response after chemoembolization of hepatocellular carcinoma. Eur J Radiol 75: e9-e14, 2010.

59. Chung JC, Naik NK, Lewandowski RJ, Deng J, Mulcahy MF, Kulik LM, Sato KT, Ryu RK, Salem R, Larson AC, et al: Diffusion-weighted magnetic resonance imaging to predict response of hepatocellular carcinoma to chemoembolization. World J Gastroenterol 16: 3161-3167, 2010.
60. Kubota K, Yamanishi T, Itoh S, Murata Y, Miyatake K, Yasunami H, Morio K, Hamada N, Nishioka A and Ogawa Y: Role of diffusion-weighted imaging in evaluating therapeutic efficacy after transcatheter arterial chemoembolization for hepatocellular carcinoma. Oncol Rep 24: 727-732, 2010.

61. Bonekamp S, Jolepalem P, Lazo M, Gulsun MA, Kiraly AP and Kamel IR: Hepatocellular carcinoma: Response to TACE assessed with semiautomated volumetric and functional analysis of diffusion-weighted and contrast-enhanced MR imaging data. Radiology 260: 752-761, 2011.

62. Sahin H, Harman M, Cinar C, Bozkaya H, Parildar M and Elmas N: Evaluation of treatment response of chemoembolization in hepatocellular carcinoma with diffusion-weighted imaging on 3.0-T MR imaging. J Vasc Interv Radiol 23: 241-247, 2012.

63. Vandecaveye V, Michielsen K, De Keyzer F, Laleman W, Komuta M, Op de beeck K, Roskams T, Nevens F, Verslype C and Maleux G: Chemoembolization for hepatocellular carcinoma: 1-month response determined with apparent diffusion coefficient is an independent predictor of outcome. Radiology 270: 747-757, 2014.

64. Vouche M, Salem R, Lewandowski RJ and Miller FH: Can volumetric ADC measurement help predict response to Y90 radioembolization in HCC? Abdom Imaging 40: 1471-1480, 2015.

65. Mannelli L, Kim S, Hajdu CH, Babb JS and Taouli B: Serial diffusion-weighted MRI in patients with hepatocellular carcinoma: Prediction and assessment of response to transarterial chemoembolization. Preliminary experience. Eur J Radiol 82: 577-582, 2013.

66. Yuan Z, Li WT and Peng WJ: Pre-treatment apparent diffusion coefficient is imaging biomarker for prediction of response to chemoembolization in hepatocellular carcinoma. Eur J Radiol 82: e901-e902, 2013

67. Dong S, Ye XD, Yuan Z, Xu LC and Xiao XS: Relationship of apparent diffusion coefficient to survival for patients with unresectable primary hepatocellular carcinoma after chemoembolization. Eur J Radiol 81: 472-477, 2012.

68. Corona-Villalobos CP, Halappa VG, Bonekamp S, Eng J, Reyes D, Cosgrove D, Rastegar N, Pan L, Pawlik TM and Kamel IR: Functional magnetic resonance imaging response of targeted tumor burden and its impact on survival in patients with hepatocellular carcinoma. Invest Radiol 50: 283-289, 2015.

69. Ye XD, Li WT and Yuan Z: Apparent diffusion coefficients at diffusion-weighted MR imaging: Potential predictors of survival in patients with hepatocellular carcinoma treated with chemoembolization. Radiology 272: 920-921, 2014

70. Mori Y, Tamai H, Shingaki N, Moribata K, Deguchi H, Ueda K, Inoue I, Maekita T, Iguchi M, Kato J, et al: Signal intensity of small hepatocellular carcinoma on apparent diffusion coefficient mapping and outcome after radiofrequency ablation. Hepatol Res 45: 75-87, 2015.

71. Ye XD, Li WT and Yuan Z: Is volumetric functional MR imaging superior to current anatomic imaging response criteria for hepatocellular carcinoma after intraarterial therapy? Radiology 271 : 619-620, 2014

72. Zhang Y,Zhao J, Guo D, Zhong W and Ran L: Evaluation of shortterm response of high intensity focused ultrasound ablation for primary hepatic carcinoma: Utility of contrast-enhanced MRI and diffusion-weighted imaging. Eur J Radiol 79: 347-352, 2011.

73. Padhani AR, Liu G, Koh DM, Chenevert TL, Thoeny HC, Takahara T, Dzik-Jurasz A, Ross BD, Van Cauteren M, Collins D, et al: Diffusion-weighted magnetic resonance imaging as a cancer biomarker: Consensus and recommendations. Neoplasia 11: 102-125, 2009.

74. Park YS, Lee CH, Kim JH, Kim IS, Kiefer B, Seo TS, Kim KA and Park CM: Using intravoxel incoherent motion (IVIM) MR imaging to predict lipiodol uptake in patients with hepatocellular carcinoma following transcatheter arterial chemoembolization: A preliminary result. Magn Reson Imaging 32: 638-646, 2014.

75. Koh DM: Science to practice: Can intravoxel incoherent motion diffusion-weighted MR imaging be used to assess tumor response to antivascular drugs? Radiology 272: 307-308, 2014.

76. Yuan Z, Zhang J, Yang H, Ye XD, Xu LC and Li WT: Diffusionweighted MR imaging of hepatocellular carcinoma: Current value in clinical evaluation of tumor response to locoregional treatment. J Vasc Interv Radiol 27: 20-30, 2016.

77. Hiraoka A, Hirooka M, Ochi H, Koizumi Y, Shimizu Y, Shiraishi A, Yamago H, Tanihira T, Miyata H, Ninomiya T, et al: Importance of screening for synchronous malignant neoplasms in patients with hepatocellular carcinoma: Impact of FDG PET/ CT. Liver Int 33: 1085-1091, 2013. 
78. Shiomi S and Kawabe J: Clinical applications of positron emission tomography in hepatic tumors. Hepatol Res 41: 611-617, 2011.

79. Lee JW, Yun M, Cho A, Han KH, Kim DY, Lee SM and Lee JD: The predictive value of metabolic tumor volume on FDG PET/ CT for transarterial chemoembolization and transarterial chemotherapy infusion in hepatocellular carcinoma patients without extrahepatic metastasis. Ann Nucl Med 29: 400-408, 2015.

80. Lee JW, Oh JK, Chung YA, Na SJ, Hyun SH, Hong IK, Eo JS Song BI, Kim TS, Kim do Y, et al: Prognostic significance of ${ }^{18}$ F-FDG uptake in hepatocellular carcinoma treated with transarterial chemoembolization or concurrent chemoradiotherapy: A multicenter retrospective cohort study. J Nucl Med 57: 509-516, 2016.

81. Hartenbach M, Weber S, Albert NL, Hartenbach S, Hirtl A, Zacherl MJ, Paprottka PM, Tiling R, Bartenstein P, Hacker M, et al: Evaluating treatment response of radioembolization in intermediate-stage hepatocellular carcinoma patients using ${ }^{18}$ F-Fluoroethylcholine PET/CT. J Nucl Med 56: 1661-1666, 2015.

82. Jo IY, Son SH, Kim M, Sung SY, Won YK, Kang HJ, Lee SJ, Chung YA, Oh JK and Kay CS: Prognostic value of pretreatment ${ }^{18}$ F-FDG PET-CT in radiotherapy for patients with hepatocellular carcinoma. Radiat Oncol J 33: 179-187, 2015.

83. Cho E, Jun CH, Kim BS, Son DJ, Choi WS and Choi SK: 18F-FDG PET CT as a prognostic factor in hepatocellular carcinoma. Turk J Gastroenterol 26: 344-350, 2015.

84. Ma W, Jia J, Wang S, Bai W, Yi J, Bai M, Quan Z, Yin Z, Fan D, Wang J, et al: The prognostic value of ${ }^{18} \mathrm{~F}-\mathrm{FDG}$ PET/CT for hepatocellular carcinoma treated with transarterial chemoembolization (TACE). Theranostics 4: 736-744, 2014

85. Young H, Baum R, Cremerius U, Herholz K, Hoekstra O, Lammertsma AA, Pruim J and Price P; European Organization for Research and Treatment of Cancer (EORTC) PET Study Group: Measurement of clinical and subclinical tumour response using $\left[{ }^{18} \mathrm{~F}\right]$-fluorodeoxyglucose and positron emission tomography: Review and 1999 EORTC recommendations. Eur J Cancer 35: 1773-1782, 1999.

86. Wahl RL, Jacene H, Kasamon Y and Lodge MA: From RECIST to PERCIST: Evolving Considerations for PET response criteria in solid tumors. J Nucl Med 50 (Suppl 1): 122S-150S, 2009.

87. Kuang Y, Salem N, Tian H, Kolthammer JA, Corn DJ, Wu C, Wang F, Wang Y and Lee Z: Imaging lipid synthesis in hepatocellular carcinoma with [methyl- $\left.{ }^{11} \mathrm{c}\right]$ choline: Correlation with in vivo metabolic studies. J Nucl Med 52: 98-106, 2011.

88. Chen CY, Li CW, Kuo YT, Jaw TS, Wu DK, Jao JC, Hsu JS and Liu GC: Early response of hepatocellular carcinoma to transcatheter arterial chemoembolization: Choline levels and MR diffusion constants - initial experience. Radiology 239: 448-456, 2006.
89. Kuo YT, Li CW, Chen CY, Jao J, Wu DK and Liu GC: In vivo proton magnetic resonance spectroscopy of large focal hepatic lesions and metabolite change of hepatocellular carcinoma before and after transcatheter arterial chemoembolization using 3.0-T MR scanner. J Magn Reson Imaging 19: 598-604, 2004.

90. Wu B, Peng WJ, Wang PJ, Gu YJ, Li WT, Zhou LP, Tang F and Zhong GM: In vivo $1 \mathrm{H}$ magnetic resonance spectroscopy in evaluation of hepatocellular carcinoma and its early response to transcatheter arterial chemoembolization. Chin Med Sci J 21: 258-264, 2006.

91. Bian DJ, Xiao EH, Hu DX, Chen XY, Situ WJ, Yuan SW, Sun JL and Yang LP: Magnetic resonance spectroscopy on hepatocellular carcinoma after transcatheter arterial chemoembolization. Chin J Cancer 29: 198-201, 2010.

92. Schilling A, Gewiese B, Berger G, Boese-Landgraf J, Fobbe F, Stiller D, Gallkowski U and Wolf KJ: Liver tumors: Follow-up with P-31 MR spectroscopy after local chemotherapy and chemoembolization. Radiology 182: 887-890, 1992.

93. Meyerhoff DJ, Karczmar GS, Valone F, Venook A, Matson GB and Weiner MW: Hepatic cancers and their response to chemoembolization therapy. Quantitative image-guided 31P magnetic resonance spectroscopy. Invest Radiol 27: 456-464, 1992.

94. Yuan Z, Ye XD, Dong S, Xu LC and Xiao XS: Evaluation of early imaging response after chemoembolization of hepatocellular carcinoma by phosphorus-31 magnetic resonance spectroscopyinitial experience. J Vasc Interv Radiol 22: 1166-1173, 2011.

95. Yuan Z, Li WT, Ye XD, Zhu HY and Peng WJ: Novel functional magnetic resonance imaging biomarkers for assessing response to therapy in hepatocellular carcinoma. Clin Transl Oncol 16: 599-605, 2014.

96. Donati OF, Hany TF, Reiner CS, von Schulthess GK, Marincek B, Seifert B and Weishaupt D: Value of retrospective fusion of PET and MR images in detection of hepatic metastases: Comparison with ${ }^{18} \mathrm{~F}-\mathrm{FDG}$ PET/CT and Gd-EOB-DTPA-enhanced MRI. J Nucl Med 51: 692-699, 2010.

97. Beiderwellen K, Gomez B, Buchbender C, Hartung V, Poeppel TD, Nensa F, Kuehl H, Bockisch A and Lauenstein TC: Depiction and characterization of liver lesions in whole body $\left[{ }^{18} \mathrm{~F}\right]-F D G$ PET/MRI. Eur J Radiol 82: e669-e675, 2013.

98. Catalano OA, Rosen BR, Sahani DV, Hahn PF, Guimaraes AR, Vangel MG, Nicolai E, Soricelli A and Salvatore M: Clinical impact of PET/MR imaging in patients with cancer undergoing same-day PET/CT: Initial experience in 134 patients: a hypothesis-generating exploratory study. Radiology 269: 857-869, 2013.

99. Nielsen K, Scheffer HJ, Pieters IC, van Tilborg AA, van Waesberghe JH, Oprea-Lager DE, Meijerink MR, Kazemier G, Hoekstra OS, Schreurs HW, et al: The use of PET-MRI in the follow-up after radiofrequency- and microwave ablation of colorectal liver metastases. BMC Med Imaging 14: $27,2014$. 